\title{
Canadian Association of Neuropathologists Abstracts of Papers Presented at the 30th Annual Meeting
}

October 5th - 7th, 1990

Quebec City, Quebec

\begin{abstract}
The 30th annual meeting of the Canadian Association of Neuropathologists was held from October 5th to 7th 1990, at the Hotel Des Gouverneurs in Quebec City, Quebec, hosted by Dr. Jacques Thibault.

The scientific sessions included 19 diagnostic cases, 17 platform presentations and 3 poster presentations. The Jerzy Olszewski guest lecture was given by Dr. Vincent Raymond of Quebec City, entitled "Psychiatric Genetics: The Search for the Susceptibility Genes for Schizophrenia and Bipolar Affective Disorders". The Royal College of Physicians and Surgeons of Canada speaker was Dr. John W. Olney of St. Louis, Missouri, and his talk was entitled "Excitatory Neurotransmitters and Neuropsychiatric Disorders".

Two awards were given for the best papers presented by trainees or residents. The Morrison H. Finlayson Award went to Michael S. Pollanen of Toronto, and the Mary Tom Award went to Dr. Sylvia Gaytan-Garcia of London, Ontario. An honorable mention was also given to Dr. Robert Hammond of London, Ontario.
\end{abstract}




\section{Abstracts of Papers Presented at the 30th Annual Meeting of the Canadian Association of Neuropathologists}

1.

\section{Senile Plaques in Parkinsonism-dementia Complex on Guam}

\section{H. ITO, A. HIRANO and S.-H. YEN (Bronx, U.S.A.)}

The parkinsonism-dementia complex (PDC) on Guam is characterized histopathologically by neuronal loss in widespread areas and by the presence of numerous Alzheimer's neurofibrillary tangles in certain neuron groups. It has been reported that in Guamanian PDC patients there is virtually no plaque formation. Prior to 1965, the existence of argentophilic plaques has been investigated in only a few Guamanian cases. However, from more recent studies, there is evidence on plaque formation in a relatively high proportion of Guamanian patients. To determine whether this change is due to differences in the detection techniques, we have re-evaluated with an immunoperoxidase method the archival material used in earlier studies.

Brain sections from 10 PDC cases were investigated. Five cases with clinico-pathologically proven Alzheimer's disease were included for comparison. Two PDC cases (died in 1962 and 1978) were shown by the modified Bielschowksy method to contain numerous plaques $(\mathrm{P}+)$; two others (died in 1963 and 1964) had few plaques (P \pm ) and in the remaining 6 cases (died in 1963-1964) no plaques (P-) could be detected with any histochemical method. Sections of hippocampus, entorhinal cortex and adjacent temporal cortex were stained immunohistochemically after formic acid pretreatment for $\beta$-amyloid protein.

Numerous deposits of reaction products were seen in both $\mathrm{P}+$ cases. The number and the distribution of the subtypes of plaques were indistinguishable from those of Alzheimer's disease. It is to be noted that one of the P+ cases had amyloid angiopathy. A small number of diffuse plaques scattered in the entorhinal-temporal cortex and very rare neuritic plaques were observed in the $\mathrm{P} \pm$ cases. The hippocampi of these patients had numerous tangles as identified with the modified Bielschowsky stain, but only few plaques could be detected immunohistochemically. No $\beta$-amyloid deposition was seen in all six Pcases. These observations corroborate the conclusions reached in earlier studies with respect to the absence of senile plaques in PDC patients.

Supported in part by: The Amyotrophic Lateral Sclerosis Association

2.

A Line of Plaques in Area 17 of Alzheimer's Disease: Relationship with Afferent Fibres and $\beta$-Amyloid Protein Distribution

THOMAS G. BEACH and EDITH G. MCGEER (Vancouver, British Columbia)

We have previously reported the presence of a distinctive line of plaques in area 17 of Alzheimer's disease (AD) brain
(Beach, TG, McGeer, EG, Brain Res 1988; 463: 357-361). Quantification of plaque numbers along lines drawn parallel to the cortical laminae confirms that there is a significant aggregation of plaques along the interface of layers IVc and V. Since current theories postulate that plaques are formed from the degeneration of specific axonal ramifications, and that $\mathrm{AD}$ "spreads" along neuroanatomical connections, an investigation of the afferent systems adjacent to the line of plaques was performed. Cholinergic axons in layers IVc and V were stained using acetylcholinesterase histochemistry. Pyramidal neuron axons in the same layers were anterogradely labelled following diI crystal placement in layers II and III (Burkhalter, A, Bernardo, KL, Proc Natl Acad Sci U.S.A. 1989; 86: 10711075). The geniculocalcarine projection, ending mainly in layer IVc, was assessed using cytochrome oxidase histochemistry. Only the cholinergic system showed significant changes in AD. There was a marked depletion of AChE-positive fibres; remaining fibres displayed bulbous swellings, some of which were in close association with senile plaques. Sprouting of nerve fibres, or altered distributions of nerve fibres, were not observed. The distribution of $\beta$-amyloid protein deposition in area 17 was found to differ from that of Bielschowsky-stained plaques. There was a preferential immunolocalization of amyloid in layers I and IVc, which was not apparent in the Bielschowsky preparations. This laminar distribution matches that of cholinergic fibres within area 17 , further implicating the cholinergic system in AD. It is hypothesized that the line of plaques may form as amyloid, produced from degenerating cholinergic fibres, which diffuses from layer IVc, which is postulated to be relatively inefficient at making "classical" plaques, into layer V, which readily forms such plaques. The neuropil properties responsible for classical plaque production remain unknown, but may be related to either pyramidal neurons or microglia.

\section{3.}

\section{Bulk Isolation of Cortical Lewy Bodies}

\section{M.S. POLLANEN, C. BERGERON, L. WEYER and S. RAMJOHN (Toronto, Ontario)}

Isolation and characterization of the constitutive polypeptides of the Lewy body has been hampered by the inability to obtain tissue rich in Lewy bodies. Recently, however, Lewy bodies have been described in the cerebral cortex of some cases of dementia, providing an abundant and accessible source for extraction and analysis. We describe a procedure to produce enriched fractions of Lewy bodies by discontinuous gradient centrifugation, with enzymatic and salt extraction of cerebral homogenates. Lewy bodies isolated under these conditions maintained their conformation and histochemical properties and were ubiquitinated. Ultrastructurally, isolated Lewy bodies were composed of 8-10 nm straight unpaired filaments. Enriched fractions of Lewy bodies were largely free of copurifying neurofibrillary tangles/paired helical filaments as the majority of 
tangles, unlike Lewy bodies, did not migrate through high molar concentrations of sucrose during centrifugation. We conclude Lewy bodies and neurofibrillary tangles have distinct structural and physiochemical properties. Solubilization and electrophoretic studies are in progress.

4.

\section{A Case of Alzheimer-Type Changes in Infantile Hallervorden-Spatz Disease}

\section{L.C. ANG and C.L. VOLL (Saskatoon, Saskatchewan)}

While the clinical presentation of Hallervorden-Spatz disease (HD) is variable, its pathology is well characterized by neuronal loss, gliosis, neuroaxonal swelling and pigmentation in the globus pallidus and pars reticularis in substantia nigra. Almost all cases are familial and most patients are afflicted in the first two decades. A handful of cases have neurofibrillary tangles (NFT) in the neocortex and hippocampus (Wisniewski et al. 1979; Hartmann et al. 1983; Eidelberg et al. 1987; GaytanGarcia et al. 1990). Most reported cases are of adult onset. Except for one case (Hartmann et al. 1983), senile plaques have not been seen and the pathology in HD is thus considered unrelated to Alzheimer's disease. We report a case of infantile onset HD with NFT and senile plaques. A 32-year-old female presented at 8 months with mental retardation. She was normal at birth. There was no family history of mental retardation or other neurologic disease. Her mental status deteriorated rapidly and by 2 years she required institutionalized care. She additionally developed progressive spastic quadraplegia and marked scoliosis. She otherwise remained relatively stable until death at 32 years following acute intestinal obstruction.

General autopsy revealed intestinal obstruction resulting from a volvulus. Macrosopically, the brain was atrophied (brain wt. $725 \mathrm{gm}$ ). There was brown pigmentation in globus pallidus and substantia nigra. Microscopically, numerous neuroaxonal spheroids and NFT were noted in the cerebral cortex and brain stem. In addition to NFT, we observed senile plaques and granulovacuolar degeneration in the hippocampal formation. The NFT exhibited immunoreactivity to antiserum against paired helical filaments (PHF). Typical changes of HD consisting of neuronal loss, gliosis, neuroaxonal swelling and pigmentation were noted in the globus pallidus and substantia nigra. In only one previously reported case of HD (Hartmann et al. 1983) have senile plaques been observed in the hippocampus and this case (like most HD with NFT pathology) had an onset later in life. Our patient is thus the first reported case of infantile onset HD with Alzheimer type changes, including presence of senile plaques.

5.

\section{Human Adrenal Medullary Brain Transplantation}

\section{N.B. REWCASTLE, K.C. PETRUK and D.G. PATRY (Calgary and Edmonton, Alberta)}

Since many patients are seeking alleviation of their parkinsonism through intracerebral tissue grafts, the pathologist will eventually be called upon to examine such grafts and to respond on the biological status of the graft and the underlying disease process to allow for proper clinical assessment. Presented is experience with two such grafts. In both instances, the operative technique is biphasic: the cavity is created in the caudate nucleus approximately 7-10 days prior to grafting on the basis that the reaction in the host tissue is optimum with endothelial proliferation and stimulation of growth factors. At death at seven months postgrafting in a 59-year-old "responder", a cavity crossed by gliovascular threads but open to the ventricle remained and only on serial sectioning was a single clump of viable vascularized adrenal cells identified but prior formalin fixation blunted the tyrosine hydroxylase (TH) and chromogranin A ( $\mathrm{CgA})$ immunocytochemical interpretation. The second graft was examined $21 / 2$ years postgrafting in a 56-year-old "nonresponder". The adrenal medullary fragments were all dead, shrunken and encapsulated by dense reactive fibrous tissue, beneath which a cystic cavity extended ventrally. Although the surgical technique is the same, the morphological findings are very different. Therefore the following is suggested in assessing graft function. Tissue must be fresh and the identified operative site isolated as a block which is carefully bisected. One-half is fixed in paraformaldehyde/picric acid for 72 hours for optimum immunocytochemical demonstration of $\mathrm{TH}, \mathrm{CgA}$ and other potential markers. For EM, a very thin slice of any identifiable tissue and adjacent host caudate should be harvested from the surface of the second block which is then fixed in formalin and plastinized or paraffinized. If medullary tissue is not readily identified grossly, both blocks can then be serially sectioned. Such an idealized approach would allow for demonstration of $\mathrm{TH}$ and $\mathrm{CgA}$ indicating functioning cells, the EM would allow for demonstration of fenestrated endothelial cells of medullary origin while the formalized half would provide standard morphology and relationship to host tissue.

\section{6.}

\section{Neonatal Glycine Encephalopathy. Neuropathological} Observations in Four Patients

\section{P. AGAMANOLIS, J.L. POTTER and G. GALLOWAY (Akron, Ohio, U.S.A.)}

We studied four patients with neonatal glycine encephalopathy (GE). Three of them, who died in the newborn period, had spongy myelinopathy involving all myelinated white matter but more severely the cerebellum, corticospinal tracts and optic nerves and tracts. All these patients also had anoxic encephalopathy. The fourth patient, a sibling of one of the three others, survived severe neonatal GE and died, severely retarded, at 17 years of age. He too had spongy myelinopathy which was similar in severity and distribution to the neonatal patients. In addition, the 17-year-old patient had severe cerebellar atrophy associated with diffuse deposition of oxalate crystals in the cerebellum. The cerebellum was the site of the highest glycine content within the CNS. Cerebellar degeneration or oxalate crystal deposition have not been previously described in GE.

The findings suggest that: a) oxalate crystals are a product of glycine catabolism via glyoxylate. b) oxalate crystals deposited over many years damage the cerebellum. c) the spongy myelinopathy of glycine encephalopathy is not a progressive disorder and does not cause the neurological abnormalities. 
The neurological dysfunction of GE has been attributed to excessive synaptic inhibition. Recent experimental data suggest that glycine activates the N-methyl-D-aspartate (NMDA) receptor, thereby indirectly potentiating the action of the excitatory amino acid glutamate which binds to the NMDA receptor. This action of glycine explains neurological manifestations of an excitatory nature, particularly intractable seizures, which are an important feature of GE.

\section{7.}

\section{Brain-Behaviour Relationships in Cerebral Ischemia} Treated with MK-801

\section{R.N. AUER, M.R. ROD and I.Q. WHISHAW (Calgary and Lethbridge, Alberta)}

The objective of the present study was to determine if the degree of ischemic brain damage correlates with the degree of neurobehavioral deficit in rats. Because of concern over the efficacy of the NMDA antagonist MK-801 in treating cerebral ischemia, the second hypothesis to be tested by this study was that MK-801, given after blood supply is restored to the brain, is efficacious in reducing normothermic ischemic brain damage.

Three groups of male Wistar rats were used. The MK-801 group received $10^{1 / 2}$ min forebrain ischemia induced by carotid clamping and hypotension, followed by $5 \mathrm{mg} / \mathrm{kg}$ MK-801 iv 20 minutes postischemia. The ischemic group received the same insult but no postischemic treatment. Cranial hypothermia in both of the ischemic groups was avoided by overhead lamps, heating the head as required to maintain constant temporalis muscle temperature. A third, sham group was subjected to operation but neither ischemia nor treatment with MK-801:

In a modified Morris water maze task, animals treated with MK-801 performed better than untreated ischemic controls, but poorer than sham operated animals. Subsequent neuropathology revealed that damage was most severe in the untreated ischemic control animals, and less severe in the MK-801 treated animals. Sham operated animals showed no neuronal necrosis.

The treatment effect of MK-801 was less robust on neurobehavioral performance than on neuropathology. Analysis of individual animal performance revealed the cause to be a dissociation of neurobehavioral performance from the degree of histologic damage. A wide range of performance was possible at a given degree of brain damage, indicating additional variation at the behavioral level which is multiplied on top of the variation seen at the structural level of brain examination.

The above findings imply that clinical stroke trails may have to factor in a greater variation in neural performance than in structural brain damage, even when effective treatments are tested. Thus, larger numbers of patients will be required in comparison with laboratory animal studies, in order to demonstrate effectiveness.

\section{8.}

\section{The Epidemiology of Brain Tumors in Canada: 1959-1987}

\section{J. MAGUIRE, L.E. BECKER and Y. MAO (Toronto, Ontario)}

In this national epidemiologic study of brain tumors in Canada, we examined the geographic and temporal patterns of incidence, mortality and hospital admissions. Data sources included the National Cancer Registration System, Canada Mortality Database, Hospital Morbidity Database and the Health Information Centre of Statistics Canada. The incidence of brain tumors increased from 1969. This increase occurred mainly in the elderly age group ( 65 years + ) and was most rapid during the period 1976-1987. Risk for the development of a brain neoplasm was approximately $25 \%$ greater for men in all age groups up to age 69 years. There was a total of 23,801 deaths $(13,716$ males and 10,085 females) from brain tumors in Canada from 1959-1987. The peak age for brain tumors in both men and women increased from 55 years in 1966 to 75 years in 1985. The incidence of brain neoplasms in Canada from 1982 to 1984 remained the same in all regions with the exception of the Maritime provinces where it was approximately $25 \%$ lower. The most rapid percentage increase in the incidence of brain tumors in all age groups during the period 1971-1984 occurred in Quebec. Mortality from brain neoplasms in all age groups during the period 1985-1987 was also slightly higher in Quebec. The apparent increase in the incidence of brain neoplasms may have been influenced by improved neuro-imaging techniques from the mid-seventies. Case validation studies are currently underway to examine this possibility. The specific tumor types are also being analysed.

\section{9.}

\section{Intracranial Germ Cell Tumors in Children}

\section{FELIX and L.E. BECKER (Toronto, Ontario)}

Primary intracranial germ cell tumors are uncommon tumors. In a series of 1,474 intracranial tumors occurring in children, we identified 49 patients (3.3\%) with germ cell tumors. A striking male predominance $(80 \%)$ was present in the pineal germinomas and a female predominance $(90 \%)$ in the suprasellar germinomas. The majority (53\%) of germ cell tumors was located in the pineal region. Thirty-seven per cent were in the suprasellar region and $10 \%$ were present at both sites. Headache was the most common symptom in children regardless of tumor location. Visual disturbances were present in children with tumor at either site but the nature of the problem was different: Parinaud's syndrome occurring in tumors of the pineal. Diabetes insipidus was frequently associated with suprasellar tumors. Conventional histology (49 cases), immunohistochemistry (20 cases), and electron microscopy (10 cases) were reviewed. Histologically, $65 \%$ were germinomas, $27 \%$ were non-germinomatous and $8 \%$ were mixed. Of the non-germinomatous tumors, there were 8 teratomas, 3 endodermal sinus, and 2 choriocarcinomas. Placental alkaline phosphatase was positive in all 20 germinomas. Human chorionic gonadotropin was identified in 7 cases and alpha fetoprotein in 4 cases. Essentially negative staining was observed with antisera to glial fibrillary acidic protein, cytokeratin, desmin, and vimentin. Electron microscopy was helpful in confirming the diagnosis in endodermal sinus and mixed tumors. Based on follow-up data on 30 patients, five-year survival was $63 \%$ for all patients with germ cell tumor. Of those patients who died, tumor type included endodermal sinus tumor $(3 / 3)$, choriocarcinoma (2/2), germinoma (3/18) and teratoma $(3 / 7)$. Because of the high incidence of non-germinomatous and 
mixed germ cell tumors, biopsy of these tumors is important in order to effectively target therapy. The correct identification of mixed germ cell tumors requires adequate tumor sampling not always readily achieved by stereotactic biopsy.

\section{0.}

\section{Immunohistochemistry and Electron Microscopy of Glomeruloid Vascular Structures in Glioblastoma Multiforme}

\section{A. M. ROJIANI and K. DOROVINI-ZIS (Vancouver, British Columbia)}

Microvascular proliferation and glomeruloid vascular structures (GVS) are an important histopathological feature of glioblastoma multiforme. The nature of cells participating in the formation of these structures remains unclear and forms the subject of this study. Immunohistochemical markers directed against Factor VIII related antigen (FVIII), alpha-smooth muscle actin (SMA) and the lectin Ulex Europaeus agglutinin (ULEX) were used. Double chromogen studies using GFAP and one of the above were also performed. Positive staining with FVIII and ULEX was seen on cells lining vascular channels. Additionally, many peripheral cells in the GVS were also positive with these markers. SMA positivity was seen in smooth muscle cells of large and small vessels, however, some endothelial cells, particularly those lining microchannels, also stained positively.

Ultrastructurally, interendothelial tight junctions and Weibel Palade bodies were seen. Cells at the periphery of GVS, stained positively with SMA, but did not possess the cytoskeleton of smooth muscle cells. Endothelial cells were hyperplastic, often overlapping each other and protruding into the lumen. They showed marked variability in size, shape and content of cytoplasmic organelles including Weibel Palade bodies. Many minute lumina were observed, which were not appreciated at light microscopic level. Pericyte-like cells were often present in abluminal locations, embedded within abnormally thickened basal lamina.

Our studies indicate that glomeruloid vascular structures in glioblastoma multiforme are complex aggregates of microchannels lined by endothelial cells and incompletely supported by cells closely resembling pericytes.

Supported by: NIH grant R01NS23746 and MRC Canada grant MA 9823

11.

\section{Gangliogliomas Associated with Partial Complex Seizures}

\section{S. GAYTAN-GARCIA, J.C.E. KAUFMANN, W.T. BLUME and J.P. GIRVIN (London, Ontario)}

Gangliogliomas are rare, slow-growing lesions occurring mostly during the first three decades. In general series they represent less than $2 \%$ of all intracerebral tumours. These are a somewhat controversial group of tumours; their clinical presentation, radiological features and natural history have not been clearly defined.

This retrospective study encompasses a period of 15 years and 10 months (February 1, 1974 to December 31, 1989) during which 368 patients underwent unilateral lobectomies or corticectomies to control drug-resistant seizures. Seizure histories, ictal and interictal electroencephalographic recordings, neuroimaging and neuropsychological assessment were used to determine the epileptic focus. Neuropathological examination revealed 110 primary brain tumours $(30 \%), 14$ cases were gangliogliomas (12.7\%). All 14 patients presented with longstanding complex partial seizures (CPS) beginning at median age of 6.5 years (mean 9.8 years; range 1 month to 44 years). The median age of surgery was 19.5 years (mean 24.3 years; range from 10 to 55 years). There were 9 male and 5 female patients. One tumour was situated in the right frontal lobe, the remainder were in the temporal lobe, usually involving mesial structures. All gangliogliomas were single and relatively well circumscribed. Size varied from 0.6 to $4 \mathrm{~cm}$ in the greatest diameter. Six were calcified and 5 showed cystic degeneration. Microscopically they showed 2 components: ganglioneuromatous and gliomatous. In the ganglioneuromatous area ganglion cells were recognized as neoplastic because of cytoarchitectural abnormalities, loss of cellular lamination, loss of polarity, variation in size and shape and bi- or trinucleation. Such criteria also allow distinction from neurons enclosed within a glial tumour. The glial component in 6 cases was exclusively astrocytic, in 4 predominantly astrocytic with a small oligodendroglial component, and in 4 cases predominantly oligodendroglial with minor astrocytic component. All the tumours were well differentiated. Abundant connective tissue coursed between the neoplastic ganglion cells in 13 tumours. A prominent vascular stroma was noticed in 7 cases. Perivascular lymphocytic infiltrate was universally seen.

In $8 / 14$ cases the non-tumoural cortex showed considerable cytoarchitectural abnormalities indicative of a maldevelopmental lesion, suggesting that gangliogliomas may be a manifestation of the same process.

12.

\section{Immunohistochemical Study of Folliculo-Stellate Cells in Pituitary Lesions}

\section{H. NISHIOKA, J.F. LLENA and A. HIRANO (Bronx, U.S.A.)}

With the benefit of recent advances in immunhistochemistry, many studies regarding folliculo-stellate cells (FSC) have been reported in normal pituitary glands and pituitary adenomas. This immunohistochemical study using antibodies specific to $S-100$ protein, GFAP, vimentin, NSE and EMA includes neoplasms and various non-neoplastic lesions such as recent and old infarcts, various hypophysectomies by surgery and alcohol injection, amyloidosis, hemochromatosis and abscess.

FSC showed strong affinity to $S-100$ protein and some were, in addition, reactive to GFAP and vimentin but showed no reactivity to NSE and EMA. Most of the pituitary adenomas showed reactive FSC at the tumor margin while metastatic tumors and Rathke's cyst showed no reactive FSC. Increased immunoreactivity of FSC was observed in fibrosis secondary to hypophysectomy, amyloidosis, hyaline deposits and abscess while no reactive FSC was seen in both recent and old infarcts. The immunoreactive FSC were increased in both number and intensity and were distributed mainly at the margin of the lesions within the adenohypophysis. 
13.

\section{The Neuromuscular Pathology of the Eosinophilia Myalgia Syndrome}

\section{P. G. GALLOWAY, D.P. AGAMANOLIS and H. FRIEDMAN (Akron, Ohio, U.S.A.)}

A syndrome of myalgia and peripheral eosinophilia has been reported recently in many patients using over the counter Ltryptophan. We studied two patients who received L-tryptophan: (1) muscle and skin biopsies from a 35-year-old man who had not received any treatment before biopsy and (2) muscle and nerve biopsies from a 36-year-old woman who had received steroid therapy prior to biopsy. Muscle from case one showed a picture almost identical to childhood dermatomyositis, with perifascicular atrophy of muscle, but no endothelial tubular inclusions by ultrastructural analysis. Small vessel vasculitis was seen in muscle, perimysium and fascia. All inflammation was lymphocytic except for two foci of eosinophils in the fascia. The skin biopsy showed minimal lymphocytic infiltrates in the upper dermis. The muscle from case two showed scattered necrotic fibers, perimysial (predominantly) and endomysial inflammation and small vessel vasculitis. The inflammation was predominantly mononculear but rare eosinophils were also seen. There were also atrophic fibers and fiber type grouping suggestive of denervation. The sural nerve biopsy showed mononuclear inflammatory infiltrates in the perineurium and soft tissue between nerve fascicles. Perivascular inflammation and small vessel vasculitis were present.

The eosinophilia-myalgia syndrome associated with L-tryptophan ingestion may present a wide spectrum of changes of inflammatory neuromyopathy and vasculitis with inconspicuous eosinophil infiltrates. Clinical manifestations and pathological changes may persist and evolve despite treatment with steroids.

14.

\section{An Unusual Case of Visceral Myopathy Associated with Peripheral Neuropathy}

\section{J. PROVIAS and C. BERGERON (Toronto, Ontario)}

We present the case of a 27-year-old white male who developed an acute abdomen, secondary to perforation of a jejunal diverticulum. Subsequent investigations showed the presence of diffuse wide-mouthed diverticulae throughout the length of the small intestine as well as a severe mixed sensory motor polyneuropathy. The neuropathy had features of both axonal degeneration and segmental demyelination on pathologic examination. A male sibling, aged 29 , has been shown to have a demyelinating peripheral neuropathy. In addition, this sibling has asymptomatic multiple colonic diverticulae.

The purpose of our presentation is to highlight the neuropathologic aspects of this unusual case, specifically the changes in visceral smooth muscle and peripheral nerve. We present each of light microscopic, immunohistochemical and EM studies on both bowel smooth muscle and sural nerve.

The visceral myopathies are a heterogeneous group of diseases, usually familial, characterized by an abnormality of the smooth muscle layers of bowel wall. Gastrointestinal symptoms are usually hypomotility, pseudo-obstruction and secondary malabsorption and diverticulosis. Recently, a few cases have been described with coexisting external ophthalmoplegia and peripheral neuropathy. These cases, as well as the findings in our case, and the known presence of symptoms of GI dysfunction in some cases of otherwise classic mitochondrial syndromes, suggests that at least some forms of visceral myopathy may belong to the spectrum of mitochondrial disorders.

\section{5.}

Neuropathology of a Progressive Recessively Inherited Case of Spastic Ataxia of the Beauce Type. A Real Nosological Entity?

\section{J. MICHAUD, C. DUPUIS, M. VANASSE and}

J.-P. BOUCHARD (Montreal and Laval, Quebec)

In the frame of the Quebec cooperative study of Friedreich's ataxia, an extensive survey of the situation of hereditary ataxia in the Province of Quebec was carried out and led, among a variety of data, to a classification of hereditary ataxias, better understanding of the classical form and some associated disorders, descriptions of specific variants and discovery of geographic isolates which remain poorly understood.

We report the clinical history and morphological findings in a 23-year-old male who presented at 11-12 years of age with a picture of spastic ataxia worse in the lower limbs, slight dysarthria and slight decrease of the vibratory sense. At 16, sections of the Achilles tendons were done. The following years, Parkinson-like tremors of both hands appeared. His neurological symptoms worsened over the years and he was last admitted for a selective posterior rhizotomy. He died of massive pulmonary emboli two days after the surgery. A paternal ancestor and his sister (subsequently) had similar symptoms. Morphologically, changes of central and peripheral distal axonopathy were found in the gracilis anterior and posterior cortical spinal tracts and peripheral nervous system. The spinal ganglia showed mild to moderate neuronal loss. Mild neuronal loss was present in the dentate nuclei with mild demyelination of the hilum. Moderate to severe neuronal loss was also noted in the motor cortex, motor cranial nerve nuclei and anterior horns of the spinal cord. The substantia nigra was also moderately involved. The heart was normal.

A clinical impression of hereditary chronic spastic ataxia of the Beauce type was given and this is the first morphological study associated with such a clinical picture. Clinical data of cases examined in this geographic region will be reviewed. They further illustrate the wide variations observed in hereditary ataxias in general. Recognition of this subtype as a nosological entity awaits further clinical and morphological studies.

\section{6.}

\section{Powassan Virus Encephalitis: A Neuropathological Study}

\section{J.B. LAMARCHE and A. MERMINOD (Sherbrooke, Quebec)}

Powassan virus is a member of the tick-borne flavivirus complex (group B arbovirus). It was first isolated in man in 1958 from a fatal case of encephalitis in the town of Powassan, Ontario. Since then, a small number of mostly non-fatal human 
cases of Powassan virus encephalitis has been described in North America mainly in Ontario, Quebec and New York state and only one neuropathological study has been carried out.

We report the case of a 6-year-old boy living on a farm in the Eastern townships of the province of Quebec who died 7 days following the onset of a fulminating meningoencephalitis. This was characterized by fever, nuchal rigidity and weakness of left upper limb followed 6 days later by seizures, rapid deterioration of consciousness and signs of uncal herniation. There was no history of tick bite. The serological studies showed a conversion of hemagglutination inhibition titers to Powassan virus from $1: 20$ to $1: 160$. Cultures of cerebrospinal fluid and brain tissue were negative, but no intracerebral inoculation of suckling mice was performed.

At autopsy, the brain weighed $1670 \mathrm{~g}$ and demonstrated severe edema with evidence of uncal and diencephalic herniation. Microscopic findings included infiltration of subarachnoid and perivascular spaces by mononuclear cells, intense microglial reaction with nodule formation and focal necrosis with frequently abundant polymorphonuclear leukocytes. These changes were disseminated predominantly in the gray structures affecting most intensely the corpus striatum and brain stem especially the pons. Several lesions resembling micro-abscesses were found in the white matter. Immunoreactivity to Powassan antigen in formalin-fixed brain tissue was negative.

The neuropathological picture of this case of Powassan virus encephalitis appears essentially similar to that of any other arthropod borne acute encephalitis and differs from the original reported case by the presence of massive edema, the conspicuous involvement of the white matter and the prominent polymorphonuclear inflammatory response.

17.

Neuropathologic Findings in Patients with AIDS: A Review of 45 Patients From a Large West-Coast Urban Center

\section{J.K. HOLDEN, K. BERRY, M.C. BOYD and H.V. VINTERS (Los Angeles, U.S.A.)}

Our purpose was to review the neuropathologic findings in 45 patients with AIDS encountered at a large urban west coast medical center. The 45 patients who constituted the material for this study included 42 males and 3 females with an age range of $20-68$ years. The review included 39 autopsies, 9 surgical specimens, and material from three patients who had both a neurosurgical procedure and necropsy. Of the patients reviewed, only 7 (15.6\%) showed no neuropathological abnormality. The single most common finding was that of a low grade (microglial nodule) encephalitis, with or without the presence of giant cells characteristic of HIV infection of the nervous system - findings thought to be typical of or correlated with the clinical syndrome of AIDS dementia complex. This was seen in 15 patients $(33 \%)$. The second most common finding was that of CNS lymphoma, which was usually multifocal and extremely necrotic. Lymphoma was seen in 14 patients $(31 \%)$. Toxoplasmosis was encountered in three patients $(6.7 \%)$. Opportunistic viral infections included evidence of cytomegalovirus (CMV) encephalitis in four patients $(8.9 \%)$ and progressive multifocal leukoencephalopathy in only two patients. A single individual showed multifocal necrotic and extremely hemorrhagic abscesses sec- ondary to (widely disseminated) aspergillosis. Two patients showed an unusual myelopathy, possibly associated with HIV infection of the spinal cord or cytomegalovirus - the latter was present in the brains of both patients.

Conclusion: The pattern of neuropathologic findings differs somewhat from that reported in other large centers where many AIDS patients are encountered insofar as CNS lymphoma shows a very high prevalence in this population. By contrast, opportunistic infections (e.g. with cytomegalovirus or Toxoplasma) appeared to be less common than reported in other large studies. The reasons for these differences may be multifactorial, but the findings suggest that, in this geographic area, the most likely diagnosis of a mass lesion in a patient with AIDS is CNS lymphoma.

18.

Clinico-Neuropathologic Correlation of Silk Suture as Embolic Agent for Cerebral AVM's

\section{H.J. MANZ, J.P. DEVEIKIS, A.J. LUESSENHOP,} A.J. CAPUTY, A.I. KOBRINE, D. SCHELLINGER and N. PATRONAS (Washington, D.C., U.S.A.)

Interventional neuroradiologists have used a variety of materials for endovascular embolization of cerebral AVM's as either a definitive or adjunctive therapeutic modality. From September 1988 to May 1989, silk sutures alone or in combination with other embolic agents (Avitene, polyvinyl alcohol, 30\% ethyl alcohol in non-ionic contrast, and flow-directed barium-impregnated silicone rubber spheres) were used in 6 patients. Three of these suffered cerebral hemorrhages post-embolization, and 2 of these later died. Neuropathological material was obtained by neurosurgical resection of four patients and by necropsy on one, from 1 to 14 days post-embolization. Severe necrotizing vasculitis, marked perivascular inflammation and variable degrees of luminal thrombosis were recognized. The degree of mural and extravascular inflammation was much more extensive than in other cases embolized with agents other than silk. This high incidence of hemorrhage and death in this small series has prompted termination of the practice of using silk sutures as embolic agent for cerebral AVM's.

19.

Myeloencephalopathy Due to Intrathecal Vincristine: Report of a Case

\section{HOUDE, C. DUPUIS, A. LORTIE and J. MICHAUD}

(Montreal, Quebec)

The treatment of acute lymphoblastic leukemia, after the obtention of remission, includes intrathecal prophylactic injections of methotrexate and cytosine arabinoside along with intravenous vincristine. A case of fatal myeloencephalopathy secondary to an accidental intrathecal administration of $1 \mathrm{mg}$ of vincristine in a five-year-old boy is reported.

Following the injection, he rapidly complained of headache. A continuous lumbar drainage was performed but he rapidly developed dizziness. Nuchal rigidity, fever, opisthotonos and lower radicular signs. Three days later, hallucinations, incoher- 
ent talking and uncontrolled movements of the right hand were noted. All cranial nerve functions were normal. Deep tendon reflexes were normal in the upper extremities but abolished in the lower extremities. There was no Babinski's sign. The cerebro-spinal fluid showed numerous polymorphonuclear leukocytes but the other parameters were in the normal limits. He subsequently developed coma and died six days after the injection.

The autopsy was performed in our center upon coroner's request. Moderate cerebral oedema and uncal herniation and mild to moderate spinal and bulbar leptomeningeal fibrosis were noted grossly. At light microscopy, changes were noted mainly in the spinal cord, medulla oblongata and at the crest of cerebellar foliae. They were more severe in the lumbar level of the spinal cord. They consisted of neuronal loss and, in the remaining neurons, alterations of the Nissl substance, swelling and neurofibrillary degeneration. Numerous axonal swellings and subpial gliosis were also found. Changes in the inferior olivary nuclei and in the cerebellar cortex enhanced the superficial topography of the neuronal loss. The nerve roots showed mild axonal degeneration and demyelination.

This case represents the sixth report of fatal accidental intrathecal vincristine administration and the fourth with morphological data. In the current case, the morphological changes are more severe than in cases with a similar evolution. The presence of neurofibrillary changes is also of interest considering a survival of only six days.
20.

\section{Familial and Sporadic Minicore Myopathy}

A. LACSON, V. SANGALANG, P. CAMFIELD, J. DOOLEY and F. BOOTH (Winnipeg, Manitoba)

Minicore (multicore) myopathy is generally a slowly progressive muscle disorder with a well-known clinical heterogeneity. The inherited variety may be transmitted either by a recessive or a dominant gene. Its morphologic hallmark (i.e., minicores in all fibre types) occur in various other myopathies.

We report six cases presenting with mild weakness in childhood and one adult case associated with minicores in their muscle biopsies. Two of these are first cousins with what appears to be concurrent ichthyosis and anhydrotic (hypohydrotic) ectodermal dysplasia (probably an X-linked disorder). Two others occurred in a mother who has a strong family history of muscle disorders and her daughter suggesting an autosomal dominant form of inheritance. The genetic mechanism is unknown in the other three. Two cases have learning disabilities which may or may not be related to perinatal events, and one has mental retardation. All seven cases have had a slowly to non-progressive clinical course. None of them have a cardiomyopathy or reactions to anaesthesia.

The finding of an X-linked disorder in association with muscle weakness and minicores in the muscle biopsy suggests that in some cases, an X-linked recessive inheritance may be possible. The variable mode of inheritance almost certainly suggests that the disorder has more than one gene involvement.

\section{Titles of Diagnostic Case Presentations}

1.

Lewy Body Dementia with Superimposed Senile Changes of the Alzheimer Type

W.C. HALLIDAY AND A.A.F. SIMA (Winnipeg, Manitoba)

2.

Methanol Toxicity

S. NAJJAR, A.H. KOEPPEN AND K.D. BARRON (Albany, USA)

3.

Hepatic Encephalopathy, Diffuse and Severe, Focal Embolic Encephalomalacia, Likely Cytomegalovirus, Diffuse and Severe and Post Transplantation Lymphoma

S. GAYTAN-GARCIA, J.J. GILBERT AND C. POWER (London, Ontario)

4.

Pontine Myelinolysis due to Rapid Hypernatremia Following Hypertonic Sodium Phosphate Enema

A.G. LACSON, M. TENENBEIN AND P.M. MARKESTEYN (Winnipeg, Manitoba)

5.

Primary Cerebral Lymphoma in Type 1 Gaucher's Disease

C. GRAFFAGNINO, D. HURLBUT AND D.G. MUNOZ (London, Ontario)
6.

Central Neurocytoma

J. THIBAULT AND F. GAGNE (Quebec City, Quebec)

7.

Ganglioglioma

H.J. MANZ, R. USCINSKI, J. DEVEIKIS, AND E.E. LACK (Washington, D.C., USA)

8.

Malignant Meningioma with Hyaline Inclusions Immunoreactive for Low and High Molecular Weight Cytokeratin

E.S. JOHNSON (Edmonton, Alberta)

9.

Meningioangiomatosis with Alzheimer Neurofibrillary Tangles in Neurons

D.S. HOROUPIAN AND J. GOATES (Stanford, California, USA)

10.

GFAP-Positive Cystic Peripheral Nerve Sheath Tumor M.L. BRIDGE AND J.M. BONNIN (Indianapolis, USA)

11.

Foix-Alajouanine Syndrome

C. MACMILLAN AND J.M. BILBAO (Toronto, Ontario) 
12.

Amyloid Myopathy

J.M. BILBAO (Toronto, Ontrio)

13.

Muscle Inflammation Secondary to Immunization

M.G. NORMAN (Vancouver, British Columbia)

14.

Hemimegalencephaly

H.V. VINTERS, M.J. DE ROSA, D.L. SECOR, W.J. PEACOCK AND W.D. SHIELDS (Los Angeles, USA)

15.

Thorotrastoma (Neuroma after Thorastrast Injection)

A.H. KOEPPEN AND J.A. MARTINI (Albany, USA)

16.

Probable Slow Virus Infection in Patient with Histiocytosis "X"

M.G. NORMAN (Vancouver, British Columbia)
17.

Xanthomatous Tumor, Consistent with Xanthoma Disseminatum

R.G. HAMMOND, I.R.A. MACKENZIE, J.J. GILBERT, M.J. SHKRUM AND J.C. ENGLISH (London, Ontario)

18.

Cerebral Blastomycosis

V. JAY, N. LAPERRIERE, R.G. PERRIN (Toronto, Ontario) 19.

Whipple's Disease

R.J.B. MACAULAY AND C. BERGERON (Toronto, Ontario) 